\title{
Qualidade de cebola minimamente processada e armazenada sob refrigeração
}

\author{
Ana Carolina A. Miguel; José Fernando Durigan \\ UNESP-FCAV, Depto. Tecnologia, Via de acesso Prof. Paulo Donato Castellane, s/n, 14884-900 Jaboticabal-SP; \\ anaamiguel@yahoo.com.br; jfduri@fcav.unesp.br
}

\begin{abstract}
RESUMO
Estudou-se o efeito do armazenamento refrigerado em cebolas, na vida útil e na qualidade de seu produto minimamente processado (PMP). Cebolas 'Superex', tipo comercial, foram armazenadas em câmara fria a $10^{\circ} \mathrm{C}$ e $60 \%$ UR, por $1 ; 16$; 31; 61 e 91 dias. Nestas datas, amostras de bulbos foram minimamente processados e armazenados a $11^{\circ} \mathrm{C}$ e $61 \%$ UR. Estes produtos foram avaliados em dias alternados quanto à massa fresca e a aparência e a cada 2-3 dias quanto à coloração instrumental (luminosidade, ângulo Hue ou de cor e cromaticidade), teores de sólidos solúveis (SS) e de acidez titulável (AT) e atividade da aliinase. O armazenamento dos bulbos levou os PMP a menor perda de massa, até o agravamento da senescência, observada nos bulbos armazenados por 91 dias. Os produtos dos bulbos armazenados mantiveram aparência adequada até o $12^{\circ}-13$ dia, exceção feita aos bulbos armazenados por 91 dias que conservaram-na por apenas 9 dias. Os PMP tornaram-se mais amarelados, com ligeira redução nos teores de SS, aumento inicial nos de AT e redução na atividade da aliinase. As cebolas ‘Superex' podem ser armazenadas a $10^{\circ} \mathrm{C}$ e $60 \%$ UR por até 61 dias, com produção de produtos minimamente processados com vida útil de até 13 dias, a $11^{\circ} \mathrm{C}$ e $61 \%$ UR.
\end{abstract}

Palavras-chave: Allium cepa, processamento mínimo, pungência, vida de prateleira.

\begin{abstract}
Quality of minimally processed onions stored under refrigeration

The effect of refrigerated storage was evaluated on onion shelflife and quality of fresh-cut products. 'Superex' onions, commercial type, were stored in a cold room at $10^{\circ} \mathrm{C}$ and $60 \% \mathrm{RH}$ for 1 ; 16 ; 31 ; 61, and 91 days. During these days, samples of bulbs were minimally processed and stored at $11^{\circ} \mathrm{C}$ and $61 \% \mathrm{RH}$. These products were evaluated on alternated days, for loss of fresh mass and appearance and every 2-3 days for color (luminosity, Hue angle, and chromaticity), soluble solids (SS) and titratable acidy (TA) contents and alliinase activity. Bulbs storage reduced the fresh-cut weight loss during storage, until the senescence aggravating, observed in bulbs stored for 91 days. Minimally processed products maintained appropriate appearance until $12^{\text {th }}-13^{\text {th }}$ day, but in products produced with bulbs stored for 91 days, this time was 9 days. During this time, fresh-cut products turned to yellow, with decrease in SS contents, increase in TA, and reduction in aliinase activity. During the storage, bulbs increased the aliinase activity. Onions 'Superex' may be stored at $10^{\circ} \mathrm{C}$ and $60 \% \mathrm{RH}$ during 61 days to make minimally processed products, which have 13 days of shelf life at $11^{\circ} \mathrm{C}$ and $61 \% \mathrm{RH}$.
\end{abstract}

Keywords: Allium cepa, minimally processed, pungency, shelf-life.

(Recebido para publicação em 8 de maio de 2006; aceito em 2 de agosto de 2007)

A cebola (Allium cepa L.) é uma das hortaliças que têm sido utilizadas em larga escala no país, tanto na forma processada (pasta, molhos, picles), quanto minimamente processada (descascada, cortada em rodelas e cubos). $\mathrm{O}$ mercado para produtos à base de cebola tem experimentado crescimento significativo (Moretti \& Durigan, 2002).

A produção brasileira está em torno de 1,15 milhões de toneladas por ano, em uma área de aproximadamente 58 mil ha (Agrianual, 2005), com aumento significativo na produtividade, em todas as regiões (Ferreira, 2000). No Brasil, ela é cultivada nos estados de São Paulo (201 mil t), Santa Catarina (430 mil t), Rio Grande do Sul (156 mil t), Paraná (80 mil t) e na região Nordeste (224 mil t) (Agrianual, 2005).
O consumo per capita desta olerácea no mundo, tem variado pouco nos últimos 50 anos. No Brasil, o consumo anual por habitante é de $6 \mathrm{~kg}$ e tem se mantido relativamente estável nos últimos 20 anos. Este consumo pode ser considerado relativamente baixo e é atribuído, em parte, à pungência que varia de moderada a alta nos bulbos das cultivares brasileiras, resultado do tipo de cebola plantada, do solo e do manejo (Oliveira, 2004).

A disseminação do uso da cebola tem levado à necessidade do desenvolvimento de novos produtos a fim de suprir a demanda. O processamento mínimo dos bulbos é um método alternativo, uma vez que se propõe a preservar as características dos vegetais frescos (Cantwell, 1992). Esta opção oferece inúmeras vantagens, tais como: eliminar os inconvenientes da operação de descascamento e corte aos consumidores, ou melhor, o odor exalado e seu efeito lacrimejante; e facilitar o manuseio, permitindo que a cebola integre os mais variados produtos culinários.

O conhecimento a respeito da possibilidade de se utilizar cebolas armazenadas para o preparo destes produtos ainda é muito pequeno, pois a qualidade das mesmas pode variar com as condições e períodos de armazenamento (Kopsell \& Randle, 1992; Maia et al., 2000), interferindo nas características físico-químicas dos bulbos, e por conseguinte na qualidade do produto minimamente processado (Watada et al., 1996).

Apesar dos frutos e hortaliças minimamente processados apresentarem-se com características similares à matériaprima, os tecidos vegetais in natura, bem co no eus produtos minimamente 
processados respiram após o processamento. Estes produtos deterioram-se rapidamente, perdendo qualidade, especialmente cor e textura, como resultado da liberação de enzimas endógenas, aumento da taxa de respiração e crescimento microbiano, levando também a uma redução da vida útil do mesmo (Wiley, 1997). Estas alterações indesejáveis na qualidade são aceleradas por danos mecânicos às células, causados pelas operações de descasque e corte, o que permite o contato das enzimas com o substrato levando ao escurecimento enzimático (Durigan \& Cassaro, 2000).

Os compostos responsáveis pelo sabor, aroma e pela pungência característicos da cebola são resultantes da decomposição enzimática de precursores sem odor, os quais contém enxofre nas suas estruturas. Os bulbos intactos têm apenas leve aroma e não possuem a propriedade de irritar as mucosas do nariz e da boca, ou de provocar efeito lacrimejante. A ação do sistema enzimático ocorre ao se proceder o rompimento dos tecidos, colocando a enzima aliinase (aliina alquil-sulfonatoliase - - EC 4.4.1.4), compartimentalizada no vacúolo das células, em contato com seus substratos localizados no citoplasma (Oliveira, 2004; Randle \& Lancaster, 2002).

De acordo com Andrade \& Lima (1983), a quantidade de ácido pirúvico liberado não é diretamente afetada pela temperatura de armazenamento, mas o período de armazenamento faz com que a pungência vá se perdendo, à medida que o envelhecimento e a deterioração avançam. A diminuição da pungência ocorre devido à utilização de seus precursores como fontes de nitrogênio e enxofre para suprirem a atividade metabólica dos bulbos e o crescimento de brotos. Kopsell \& Randle (1992) afirmaram que a compreensão do comportamento da pungência ao longo do tempo de armazenamento dos bulbos permite a melhor utilização dos mesmos, tanto para consumo in natura como para processamento.

Com o objetivo de ampliar o conhecimento e atender as novas demandas do mercado por produtos minimamente processados, este trabalho se propôs a conhecer o efeito do armazenamento refrigerado de cebolas, na qualidade e vida útil de seus produtos minimamente processados.

\section{MATERIAL E MÉTODOS}

Foram utilizados bulbos de cebola da cultivar Superex (Grupo Amarela, Subgrupo Globular e Classe 2345), tipo comercial, produzidos no município de Monte Alto-SP, na safra de 2003. Os bulbos colhidos em 30/07/03 foram curados à sombra por 5 dias, destopados, padronizados quanto ao tamanho (diâmetro de 40-55 mm) e acondicionados em sacos de rede de fibra plástica, com $20 \mathrm{~kg}$ de capacidade, antes de serem armazenados em câmara fria $\left(10^{\circ} \mathrm{C}, 60 \%\right.$ UR), por até 91 dias. Durante o período de armazenamento, aos 1 (início); 16; 31; 61 e 91 dias, amostras (20 kg) foram retiradas da câmara, selecionadas e minimamente processadas sob condições assépticas.

As operações constaram de descascamento, higienização superficial com etanol a $70 \%(\mathrm{v} / \mathrm{v})$, picamento em cubos (10 mm) e enxágüe com etanol a $70 \%$ (v/v). Este material, depois de escorrido, foi embalado em bandejas de poliestireno expandido, PAC B-2F (208x140x20mm), envolvidas com filme de cloreto de polivinila (PVC), esticável e com 0,017 mm de espessura. Em cada época foram produzidas 18 embalagens contendo $300 \mathrm{~g}$ de produto, que foram armazenadas a $11^{\circ} \mathrm{C}$ e 61\% UR, simulando as condições encontradas no comércio varejista, e avaliadas em dias alternados. As variáveis analisadas foram: a) perda de massa: através de pesagem direta; b) aparência: mediante atribuição de notas (1= ótima, com pedaços túrgidos, sem podridão e coloração normal; 2= boa, sem descoloração ou perda de líquido, com sinais de hidratação; 3= regular, com pequena descoloração, sinais de hidratação e alguma perda de líquido; 4= ruim, com perda de líquido e aparecimento de podridões; $5=$ péssima, com odor desagradável e grande incidência de podridões); c) coloração: determinada com colorímetro Minolta CR 200b, e expressos em Luminosidade ( $\mathrm{L}^{*}$ ), ângulo Hue ou de cor $\left({ }^{\circ} \mathrm{h}\right)$ e cromaticidade $(\mathrm{C})$
(Minolta Corp., 1994); d) acidez titulável: determinada por titulação de $30 \mathrm{~g}$ de material triturado, homogeneizado com $50 \mathrm{~mL}$ de água destilada, com solução de $\mathrm{NaOH}$ a 0,1 mol $\mathrm{L}^{-1}$, tendo como indicador fenolftaleína a 1\%, e expressa em gramas de ácido pirúvico.100 g $\mathrm{g}^{-1}$ de polpa (Instituto Adolfo Lutz, 1985); e) sólidos solúveis: determinado por leitura direta em refratômetro digital Atago PR101, utilizando-se gotas extraídas da polpa triturada, sendo os resultados expressos em ${ }^{\circ}$ Brix (AOAC, 1997); f) pungência: determinada em duas amostras (100 g cada), sendo uma tratada termicamente em forno de microondas (potência alta, por $3 \mathrm{~min}$ ) e a outra mantida crua. Estas amostras, depois de tratadas, foram trituradas e deixadas em repouso por 5 min. Do sobrenadante, tomou-se $5 \mathrm{~g}$, que foram acrescidas de $5 \mathrm{~mL}$ de TCA a 0,5\% e agitadas por uma hora. Os extratos foram filtrados e tiveram seus volumes completados para $100 \mathrm{~mL}$, com água destilada. Reagiu-se $2 \mathrm{~mL}$ deste extrato com $1 \mathrm{~mL}$ de 2,4 dinitrofenilhidrazina a 0,0125\% dissolvida em $\mathrm{HCl}$ a $2 \mathrm{M}$. Esta reação foi incubada em banho-maria a $37^{\circ} \mathrm{C}$, por 10 min., interrompida com a adição de 5 mL de NaOH a 0,6 M, e sua absorbância lida a $420 \mathrm{~nm}$. Utilizou-se como padrão, ácido pirúvico a $2 \mathrm{mmol} \mathrm{mL}^{-1}$ e como "branco", água destilada. Os resultados foram calculados pela diferença entre os valores quantificados nos materiais, cru e aquecido, e expressos em micromoles de ácido pirúvico por grama de cebola (Schwimmer \& Weston, 1961).

Os produtos minimamente processados foram classificados quanto à pungência de acordo com o indicado pelo "VLI Sweet Index" (Vidalialabs, 2004) e quanto ao sabor pelo proposto por Miguel et al. (2004). O "VLI Sweet Index” indica que as cebolas podem ser classificadas em função da atividade da aliinase, a qual é expressa em mmoles de ácido pirúvico.g - $^{-1}$ em "muito suave" (0-2,9 mmoles $\left.\mathrm{g}^{-1}\right)$, “suave” (3,0-4,2 mmoles $\left.\mathrm{g}^{-1}\right)$, "levemente pungente" (4,3-5,5), "pungente” (5,6-6,3 mmoles $\left.\mathrm{g}^{-1}\right)$, “pungência forte" $(6,4-6,9$ mmoles $\left.\mathrm{g}^{-1}\right)$, "pungência muito forte" (7,0-7,9 mmoles $\left.\mathrm{g}^{-1}\right)$ e “picante" (8,0-10,0 mmoles $\left.\mathrm{g}^{-1}\right)$. São certificadas como “do- 
ces” as cebolas com concentração até "levemente pungente" e como "extra doce”, as com concentração até "suave”.

Miguel et al. (2004) sugerem que as cebolas podem ser classificadas mediante a combinação entre os teores de sólidos solúveis (SS) e de acidez titulável (AT) e da atividade da aliinase, indicando que uma cebola, para ter sabor considerado "picante" deve ter alta atividade da aliinase (>6,0 mmoles ác. pirúvico $\mathrm{g}^{-1}$ ), elevada acidez (>0,3 g ác. pirúvico $100 \mathrm{~g}^{-1}$ ) e baixa concentração de sólidos $\left(<6^{\circ}\right.$ Brix $)$; para sabor "moderadamente picante" deve ter valores intermediários de sólidos solúveis (6$8^{\circ}$ Brix), acidez (0,3-0,2 g ác. pirúvico $\left.100 \mathrm{~g}^{-1}\right)$ e atividade da aliinase (4,0-6,0 mmoles ác. pirúvico $\mathrm{g}^{-1}$ ); para sabor "muito pouco picante" deve apresentar níveis intermediários de atividade da aliinase (4,0-6,0 mmoles ác. pirúvico $\mathrm{g}^{-1}$ ) e de acidez (0,3-0,2 g ác. pirúvico 100 $\left.\mathrm{g}^{-1}\right)$, mas alto de sólidos solúveis ( $>9^{\circ}$ Brix); e para ser considerada "doce" deve ter alto conteúdo de sólidos solúveis ( $>9^{\circ}$ Brix), baixa a moderada acidez ( $<0,2$ g ác. pirúvico $\left.100 \mathrm{~g}^{-1}\right)$ e baixa atividade da aliinase $(<4,0$ mmoles ác. pirúvico $\mathrm{g}^{-1}$ ).

O delineamento experimental foi em blocos ao acaso com 7 a 8 tratamentos (tempo de armazenamento dos produtos minimamente processados). A evolução da perda de massa fresca, foi analisada através de análise de regressão, com as equações de $1^{\circ}$ grau comparadas quanto ao paralelismo através do teste t (Neter et al.,1978).

\section{RESULTADOS E DISCUSSÃO}

A taxa de perda de massa fresca pelos produtos minimamente processados (PMP) reduziu-se significativamente (teste t) durante o tempo de armazenamento dos bulbos (Tabela 1), variando de 0,4962\% dia ${ }^{-1}$ no PMP produzido com cebolas armazenadas por 1 dia, para até 0,2010\% dia $^{-1}$, no PMP de cebolas armazenadas por 61 dias (Tabela 1). Esta redução pode ser atribuída à perda de água livre pelos bulbos, durante o armazenamento refrigerado. Os PMP produzidos com cebolas armazenadas por 91 dias apresentaram perdas de massa equivalente à dos PMP produzidos a partir de

Tabela 1. Perda de massa fresca, representada por equações de regressão, por produtos minimamente processados de cebolas ‘Superex' armazenados a $11^{\circ} \mathrm{C}$ e $61 \%$ UR e que foram produzidos com bulbos previamente armazenados a $10^{\circ} \mathrm{C}$ e $60 \%$ UR. (Loss of fresh weight represented by regression equation, by onion 'Superex' minimally processed and stored at $11^{\circ} \mathrm{C}$ and $61 \% \mathrm{RH}$ made with bulbs previously stored at $10^{\circ} \mathrm{C}$ and $60 \% \mathrm{RH}$ ). Jaboticabal, UNESP, 2003.

\begin{tabular}{clc}
\hline Armazenamento (dia) & Equações de perda de massa fresca & Teste $\mathbf{t}$ \\
\hline 1 & $Y=-0,0250+0,4962 . X ; R=0,9999^{* *}$ & 153,1 \\
16 & $Y=0,2284+0,2848 . X ; R=0,9938^{* *}$ & 20,1 \\
31 & $Y=0,1183+0,2677 . X ; R=0,9980^{* *}$ & 38,7 \\
61 & $Y=-0,1083+0,2010 . X ; R=0,9982^{* *}$ & 40,7 \\
91 & $Y=-0,0040+0,4226 . X ; R=0,9977^{* *}$ & 38,6 \\
\hline
\end{tabular}

$\mathrm{Y}=\%$ de perda de massa; $\mathrm{X}=$ dias de armazenamento. ${ }^{* *}$ significativo a $1 \%$ de probabilidade ( $\mathrm{Y}=\%$ of fresh mass loose; $\mathrm{X}=$ days of storing; ${ }^{* *}$ significative, $1 \%$ ).

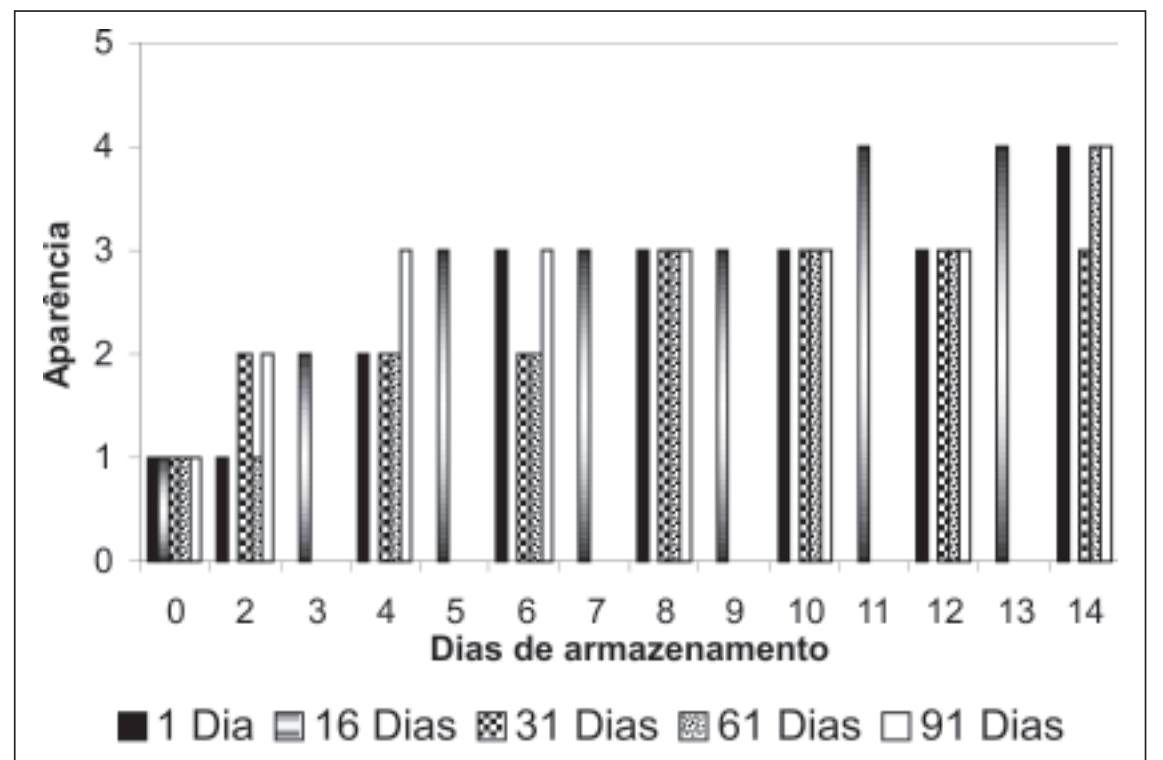

Figura 1. Aparência em produtos minimamente processados de cebolas 'Superex', armazenados a $11^{\circ} \mathrm{C}$ e $61 \%$ UR e que foram produzidos com bulbos armazenados a $10^{\circ} \mathrm{C}$ e $60 \%$ UR. (Escala: 1 = ótima; $2=$ boa; $3=$ regular; $4=$ ruim; $5=$ péssimo) (Appearence of onions 'Superex' minimally processed, stored at $11^{\circ} \mathrm{C}$ and $61 \% \mathrm{RH}$ and made with bulbs stored at $10^{\circ} \mathrm{C}$ and $60 \% \mathrm{RH}$ ). (Escala: $1=$ =xcellent; $2=$ good; $3=$ regular; $4=$ bad; $5=$ very bad) Jaboticabal, UNESP, 2003

bulbos recém-colhidos, o que pode ser atribuído ao estado de senescência destes bulbos. Os bulbos minimamente processados, produzidos com as cebolas armazenadas por um dia mantiveram aparência adequada (nota=3) até o $12^{\circ}$ dia, enquanto os produzidos com cebolas armazenadas por 16; 31 ou 61 dias, apresentaram aparência adequada até o $13^{\circ}$ dia. Os PMP produzidos com cebolas armazenadas por 91 dias, mantiveram aparência regular até o $7^{\circ}$ dia (Figura 1). Todos os produtos apresentaram perda de líquido nas embalagens após o $8^{\circ}$ dia, como o indicado por Watada et al. (1996).
A luminosidade ( $\left.\mathrm{L}^{*}\right)$ dos PMP reduziu-se ao longo do período de armazenamento, o que revela a suscetibilidade destes produtos ao escurecimento, provavelmente como conseqüência de reações enzimáticas e formação de metabólitos secundários em resposta ao corte (Role \& Chism III,1987; Burns, 1995; Chitarra, 2001). A redução nos valores do ângulo de cor (h) indica o amarelecimento destes produtos, apesar da pequena alteração na cromaticidade (Tabela 2), como o observado por Cassaro (1999), em cebolas fatiadas. 
Tabela 2. Características físicas e químicas de produtos minimamente processados de cebolas ‘Superex’ armazenados a $10^{\circ} \mathrm{C}$ e $60 \% \mathrm{UR}$ e que foram produzidos com bubos armazenados por até 91 dias, a $11^{\circ} \mathrm{C}$ e $61 \% \mathrm{UR}$. . (Physical and chemical characteristics of onions 'Superex' minimally processed, stored at $11^{\circ} \mathrm{C}$ and $61 \%$ RH and made with bulbs stored at $10^{\circ} \mathrm{C}$ and $60 \% \mathrm{RH}$ ). Jaboticabal, UNESP, 2003.

\begin{tabular}{|c|c|c|c|c|c|c|}
\hline \multirow{3}{*}{ Tempo (dia) } & \multicolumn{3}{|c|}{ Coloração } & \multirow{2}{*}{ SS ( ${ }^{\circ}$ Brix) } & \multirow{2}{*}{ AT $\left(g 100 g^{-1}\right)$} & \multirow{2}{*}{$\begin{array}{c}\text { Pungência } \\
\left(\mu \mathrm{mol} \mathrm{g} \mathbf{g}^{-1}\right)\end{array}$} \\
\hline & $\mathrm{L}^{*}$ & $\mathbf{h}^{\circ}$ & C & & & \\
\hline & \multicolumn{6}{|c|}{ Inicial (1 dia) } \\
\hline 0 & $57,2 a$ & $137,7 \mathrm{abc}$ & $2,5 \mathrm{a}$ & $9,8 a b$ & $0,130 a b$ & $4,063 \mathrm{bc}$ \\
\hline 2 & $55,2 a$ & $158,5 \mathrm{a}$ & $2,4 \mathrm{a}$ & $9,6 \mathrm{bc}$ & $0,135 a b$ & $4,327 \mathrm{abc}$ \\
\hline 4 & $51,9 a$ & $144,8 \mathrm{ab}$ & $2,5 \mathrm{a}$ & $9,6 \mathrm{bc}$ & $0,134 a b$ & $5,103 a$ \\
\hline 6 & $50,1 \mathrm{a}$ & $118,5 \mathrm{bc}$ & $2,1 \mathrm{~b}$ & $9,4 \mathrm{bc}$ & $0,136 a b$ & $4,493 a b c$ \\
\hline 8 & $54,2 \mathrm{a}$ & $118,2 b c$ & $2,0 \mathrm{~b}$ & $9,0 \mathrm{c}$ & $0,141 \mathrm{a}$ & $4,814 a b$ \\
\hline 10 & $52,7 \mathrm{a}$ & $116,9 \mathrm{bc}$ & $2,2 \mathrm{~b}$ & $9,8 a b$ & $0,127 \mathrm{~b}$ & $4,581 \mathrm{abc}$ \\
\hline 12 & $51,2 a$ & $123,1 \mathrm{abc}$ & $2,2 \mathrm{~b}$ & $10,4 \mathrm{a}$ & $0,126 \mathrm{~b}$ & $4,610 a b c$ \\
\hline 14 & $51,3 a$ & $113,1 \mathrm{c}$ & $2,3 \mathrm{~b}$ & $9,8 a b$ & $0,130 a b$ & $3,937 \mathrm{c}$ \\
\hline \multirow[t]{2}{*}{ CV (\%) } & 11,92 & 11,09 & 46,79 & 3,34 & 6,21 & 4,78 \\
\hline & \multicolumn{6}{|c|}{16 dias } \\
\hline 0 & 54,8 a & $134,8 \mathrm{ab}$ & $2,4 \mathrm{c}$ & $10,0 \mathrm{a}$ & $0,132 b$ & 5,436 a \\
\hline 3 & 55,5 a & $138,2 \mathrm{a}$ & $2,2 \mathrm{c}$ & $9,0 \mathrm{bc}$ & $0,134 \mathrm{~b}$ & $4,606 a b$ \\
\hline 5 & $45,9 a b$ & $125,1 \mathrm{bc}$ & $3,5 \mathrm{bc}$ & $9,0 \mathrm{bc}$ & $0,140 a b$ & $4,730 a b$ \\
\hline 7 & $41,6 \mathrm{~b}$ & $113,7 \mathrm{cdc}$ & $4,9 a b$ & $9,6 \mathrm{c}$ & $0,152 \mathrm{a}$ & $3,752 b c$ \\
\hline 9 & $41,5 \mathrm{~b}$ & $106,1 d$ & $5,1 a b$ & $9,2 \mathrm{bc}$ & $0,136 \mathrm{~b}$ & $5,153 \mathrm{a}$ \\
\hline 11 & $41,0 \mathrm{~b}$ & $106,7 \mathrm{dc}$ & $5,3 a b$ & $9,4 a b$ & $0,146 a b$ & $5,288 a$ \\
\hline 13 & $37,2 \mathrm{~b}$ & $105,9 \mathrm{dc}$ & $6,0 \mathrm{~b}$ & $8,8 \mathrm{bc}$ & $0,146 a b$ & $3,232 \mathrm{c}$ \\
\hline $\mathrm{CV}(\%)$ & 10,81 & 4,31 & 20,72 & 3,61 & 4,28 & 5,57 \\
\hline \multicolumn{7}{|c|}{31 dias } \\
\hline$\overline{0}$ & $61,7 \mathrm{a}$ & $143,1 \mathrm{a}$ & $2,9 a b$ & $9,0 \mathrm{a}$ & $0,133 \mathrm{bc}$ & $6,187 \mathrm{a}$ \\
\hline 2 & $57,5 a b$ & $134,8 \mathrm{ab}$ & $3,2 \mathrm{~b}$ & 9,3 a & $0,132 \mathrm{bc}$ & 5,023 a \\
\hline 4 & $55,4 a b$ & $128,7 \mathrm{bc}$ & $3,5 a b$ & $9,0 \mathrm{a}$ & $0,131 \mathrm{c}$ & $5,444 \mathrm{a}$ \\
\hline 6 & $53,2 a b$ & $130,4 \mathrm{bc}$ & $3,6 a b$ & $9,1 \mathrm{a}$ & $0,130 \mathrm{c}$ & $5,853 \mathrm{a}$ \\
\hline 8 & $47,6 a b$ & $121,7 \mathrm{~cd}$ & $3,7 a b$ & 9,3 a & $0,140 \mathrm{a}$ & $3,248 a$ \\
\hline 10 & $44,5 \mathrm{~b}$ & $118,7 \mathrm{bcd}$ & $3,9 a b$ & 9,1 a & $0,137 a b$ & 5,194 a \\
\hline 12 & $44,7 a b$ & $115,5 d$ & $3,8 \mathrm{a}$ & $9,2 \mathrm{a}$ & $0,133 \mathrm{bc}$ & 4,439 a \\
\hline 14 & $45,0 \mathrm{ab}$ & $114,6 \mathrm{~d}$ & $3,5 a b$ & $8,9 \mathrm{a}$ & $0,133 \mathrm{bc}$ & 5,493 a \\
\hline $\mathrm{CV}(\%)$ & 13,19 & 3,90 & 18,10 & 2,23 & 1,71 & 17,16 \\
\hline \multicolumn{7}{|c|}{61 dias } \\
\hline 0 & $67,6 \mathrm{a}$ & $130,1 \mathrm{a}$ & $2,8 \mathrm{~b}$ & $9,0 \mathrm{a}$ & $0,146 a b$ & $6,030 \mathrm{abc}$ \\
\hline 2 & 59,7 a & $128,2 \mathrm{a}$ & $2,7 \mathrm{~b}$ & $8,5 a b c$ & $0,138 a b$ & $5,225 b c$ \\
\hline 4 & 59,2 a & $126,5 \mathrm{a}$ & $3,7 a b$ & $9,0 a b$ & $0,131 \mathrm{~b}$ & $7,371 \mathrm{a}$ \\
\hline 6 & 57,5 a & $124,1 \mathrm{a}$ & $3,3 a b$ & $8,9 a b$ & $0,132 \mathrm{~b}$ & $5,976 \mathrm{abc}$ \\
\hline 8 & $56,8 \mathrm{a}$ & $120,1 \mathrm{a}$ & $4,9 a b$ & $9,0 a b$ & $0,129 b$ & $5,729 a b c$ \\
\hline 10 & $56,7 \mathrm{a}$ & $121,7 \mathrm{a}$ & $4,7 a b$ & $8,4 \mathrm{c}$ & $0,138 a b$ & $4,963 \mathrm{bc}$ \\
\hline 12 & $49,1 \mathrm{~b}$ & $121,2 \mathrm{a}$ & $6,4 \mathrm{a}$ & $9,1 \mathrm{a}$ & $0,132 \mathrm{~b}$ & $6,426 a b$ \\
\hline 14 & $43,3 \mathrm{~b}$ & $115,3 \mathrm{a}$ & $6,2 \mathrm{a}$ & $8,2 \mathrm{bc}$ & $0,163 \mathrm{a}$ & $4,772 \mathrm{c}$ \\
\hline CV (\%) & 11,14 & 4,08 & 21,99 & 2,48 & 3,20 & 7,14 \\
\hline \multicolumn{7}{|c|}{91 dias } \\
\hline$\overline{0}$ & $60,4 \mathrm{a}$ & $129,0 \mathrm{a}$ & $3,9 \mathrm{a}$ & $9,4 \mathrm{a}$ & $0,120 \mathrm{a}$ & $7,723 \mathrm{a}$ \\
\hline 2 & $52,9 a b$ & $127,7 \mathrm{a}$ & $3,8 \mathrm{a}$ & $9,2 a b$ & $0,143 a$ & $4,888 \mathrm{bc}$ \\
\hline 4 & $51,9 a b$ & $123,5 \mathrm{a}$ & $3,6 \mathrm{a}$ & $9,2 a b$ & $0,143 a$ & $5,657 \mathrm{abc}$ \\
\hline 6 & $50,5 a b$ & $122,9 \mathrm{a}$ & $3,4 \mathrm{a}$ & $9,2 a b$ & $0,142 \mathrm{a}$ & $6,670 a b$ \\
\hline 8 & $49,5 \mathrm{~b}$ & $119,1 \mathrm{a}$ & $3,0 \mathrm{a}$ & $8,8 \mathrm{bc}$ & 0,143 a & $5,922 \mathrm{abc}$ \\
\hline 10 & $48,4 \mathrm{~b}$ & 117,3 a & $3,1 \mathrm{a}$ & $8,4 \mathrm{c}$ & $0,151 \mathrm{a}$ & $6,217 \mathrm{abc}$ \\
\hline 12 & $48,3 \mathrm{~b}$ & $115,1 \mathrm{a}$ & $3,4 \mathrm{a}$ & $8,8 \mathrm{bc}$ & $0,142 \mathrm{a}$ & $6,633 a b$ \\
\hline 14 & $47,5 \mathrm{~b}$ & $114,1 \mathrm{a}$ & $3,2 \mathrm{a}$ & $9,2 a b$ & 0,142 a & $5,714 a b c$ \\
\hline$\overline{C V}(\%)$ & 8,89 & 6,43 & 16,81 & 2,08 & 5,70 & 9,94 \\
\hline
\end{tabular}

$\mathrm{L}^{*}=$ luminosidade; $\mathrm{h}^{\circ}$ = ângulo de cor; $\mathrm{C}$ = cromaticidade; $\mathrm{SS}$ = sólidos solúveis; $\mathrm{AT}$ = acidez titulável; pungência = atividade da aliinase, CV $(\%)=$ coeficiente de variação ( $\mathrm{L}^{*}=$ =luminosity; $\mathrm{h}^{0}=$ color angle; $\mathrm{C}=$ chromaticity; $\mathrm{SS}=$ soluble solids; AT=titratable acididy; pungency=activity of aliinase; CV (\%)=variation coefficient). 
Os conteúdos de sólidos solúveis (SS) dos PMP apresentaram leve decréscimo durante o armazenamento refrigerado, mas os de acidez titulável apresentaram algum aumento inicial, estimulado pela aceleração do metabolismo devido ao corte, seguido de redução (Tabela 2).

A pungência reduziu-se significativamente nos PMP durante o período de armazenamento e esta redução foi tanto maior quanto maior o tempo de armazenamento dos bulbos, o qual tornou os bulbos mais pungentes (Tabela 2).

Segundo a classificação indicada pelo VLI Sweet Index (Vidalialabs, 2004), os PMP produzidos com a cebola armazenada por um dia manteve-se "suave" durante seu armazenamento; os produzidos com as armazenadas por 16 dias evoluíram de "levemente picante" para "suave”, em 12 dias; os obtidos das armazenadas por 31 ou 61 dias de "pungente” para "levemente pungente”; e as dos armazenados por 91 dias de "pungência muito forte" para "picante".

Wall \& Corgan (1992) relatam que a relação entre os teores de SS, AT e pungência pode dar uma boa indicação da qualidade organoléptica, ao invés de análises isoladas destes parâmetros. Tendo-se esta condição, os produtos processados foram classificados como "muito pouco picante”, devido à pungência intermediária (4,0-6,0 mmoles ác. pirúvico $\left.\mathrm{g}^{-1}\right)$, AT intermediária $(<0,2$ g ác. pirúvico $100 \mathrm{~g}^{-1}$ ), mas alto de SS (>9Brix) (Miguel et al., 2004).

As cebolas 'Superex’ podem ser armazenadas a $10^{\circ} \mathrm{C}$ e $60 \%$ UR antes de serem utilizadas para a produção de produtos minimamente processados, por até
61 dias, com a produção de produtos com vida útil de até 12 dias, a $11^{\circ} \mathrm{C}$ e $61 \%$ UR.

\section{AGRADECIMENTOS}

Os autores agradecem à FAPESP pela bolsa de iniciação científica concedida ao primeiro autor.

\section{REFERÊNCIAS}

AGRIANUAL. 2005. Anuário da agricultura brasileira. São Paulo: FNP Consultoria \& Comércio. p. 279-282.

ANDRADE MO; LIMA UA. 1983. Cebola - produção, pré-processamento e transformação agroindustrial. In: MARTINELLI FILHO, A et al. (eds.). Processamento e tecnologia dos alimentos. Piracicaba: ESALQ/USP. p. 73-115.

AOAC. 1997. Official methods of analysis of the Association of Analytical Chemists International. Washington: AOAC. p. 37-10, 42-2, 44-3, 45-16.

BURNS JK. 1995. Lightly processed fruits and vegetables: Introduction. HortScience 30: 1415.

CANTWELL M. 1992. Postharvest handling systems: minimally processed fruits and vegetables. In: KADER, A.A. (ed). Postharvest technology of horticultural crops. Oakland: University of California. p. 277-281.

CASSARO KP. 1999. Conservação de bulbos de cebola minimamente processados. Jaboticabal: UNESP - FCAV. 77p (Monografia para conclusão de graduação).

CHITARRA MI. 2001. Alimentos minimamente processados. Lavras: UFLA/FAEPE. p. 50-52.

DURIGAN JF; CASSARO KP. 2000. Hortaliças minimamente processadas. Horticultura Brasileira 18: 159-161.

FERREIRA MD. 2000. Cultura de cebola: recomendações técnicas. Campinas: Asgrow. 36p.

INSTITUTO ADOLFO LUTZ. 1985. Normas analíticas: métodos químicos e físicos para análises de alimentos. São Paulo: IAL. 371p.

KOPSELL DE; RANDLE WM. 1992. Onion cultivars differ pungency and bulb quality changes during storage. HortScience 32: 12601263.
MAIA MCC; PEDROSA JF; TORRES FILHO J; NEGREIROS MZ; BEZERRA NETO F. 2000 Características de qualidade de cebola múltipla durante armazenamento sob condição ambiental não controlada. Horticultura Brasileira 18: 61-64.

MIGUEL ACA; DURIGAN MFB; DURIGAN JF; MORETTI CL. 2004. Postharvest quality of twelve onions cultivars grown in the southeast of Brazil. In: INTERNATIONAL ISHS SYMPOSIUM ON EDIBLE ALLIACEAE, 4. Abstracts... Beijing: ISEA. p. 235.

MINOLTA CORP. 1994. Precise color communication: color control from feeling to instrumentation. Ramsey: Minolta Corporation Instrument Systems Division. 49p.

MORETTI CL; DURIGAN FJ. 2002. Processamento de cebola. Informe Agropecuário 23: 99-104.

NETER J; WASSERMAN W; WHITMORE GA. 1978. Applied linear statistical models. Massachussetts: Alynand and Bacon. 745p.

OLIVEIRA VR. 2004. Cebola doce. Horticultura Brasileira 22: s.p. (reportagem da capa)

RANDLE WM; LANCASTER JE. 2002. Sulphur compounds in Alliums in relation to flavour quality. In: H.D. RABINOWITCH, H.D.; CURRAH, L. (Eds). Allium Crop Science: Recent Advances. p. 330-350.

ROLE RS; CHISM III GW. 1987. Physiological consequences of minimally processed fruits and vegetables. Journal of Food Quality 10: 157-177.

SCHWIMMER S, WESTON WJ. 1961. Enzymatic development of pyruvic acid in onion as a measure of pungency. Journal of Agricultural and Food Chemistry 9: 301-304.

VIDALIALABS - VLI Sweet index (Sweetometer). 2004, 7 de outubro. Disponível em http://www.vidalialabs.com/images/ sweeetometer.jpg/

WALL MM; CORGAN JN. 1992. Relationship between pyruvate analysis and flavor perception for pungency determination. HortScience 27: 1029-1030.

WATADA AE, KO NP, MINOTT DA. 1996. Factors affecting quality of fresh-cut horticultural crops. Postharvest Biology and Technology 9: 115-125.

WILEY RC. 1997. Frutas y Hortalizas Mínimamente Procesadas y Refrigeradas. Zaragoza: Acribia. 362p. 